\title{
VISCOSITY SOLUTION OF LINEAR REGULATOR QUADRATIC FOR DEGENERATE DIFFUSIONS
}

MD. AZIZUL BATEN

Received 28 May 2005; Revised 30 September 2005; Accepted 19 November 2005

The paper studied a linear regulator quadratic control problem for degenerate HamiltonJacobi-Bellman (HJB) equation. We showed the existence of viscosity properties and established a unique viscosity solution of the degenerate HJB equation associated with this problem by the technique of viscosity solutions.

Copyright (c) $2006 \mathrm{Md}$. Azizul Baten. This is an open access article distributed under the Creative Commons Attribution License, which permits unrestricted use, distribution, and reproduction in any medium, provided the original work is properly cited.

\section{Introduction}

We are concerned with the linear regulator quadratic control problem to minimize the expected cost with discount factor $\alpha>0$ :

$$
J(c)=E\left[\int_{0}^{\infty} e^{-\beta t}\left\{h\left(x_{t}\right)+\left|c_{t}\right|^{2}\right\} d t\right]
$$

over $c \in \mathscr{A}$ subject to the degenerate stochastic differential equation

$$
d x_{t}=\left[A x_{t}+c_{t}\right] d t+\sigma x_{t} d w_{t}, \quad x_{0}=x \in \mathbb{R}, t \geq 0
$$

for nonzero constants $A, \sigma \neq 0$, and a continuous function $h$ on $\mathbb{R}$, where $w_{t}$ is a onedimensinal standard Brownian motion on a complete probability space $(\Omega, \mathscr{F}, P)$ endowed with the natural filtration $\mathscr{F}_{t}$ generated by $\sigma\left(w_{s}, s \leq t\right)$, and $\mathscr{A}$ denotes the class of all $\mathscr{F}_{t}$ - progressively measurable processes $c=\left(c_{t}\right)$ with $J(c)<\infty$.

This kind of stochastic control problem has been studied by many authors [3, 7] for nondegenerate diffusions to (1.1) and (1.2). We also assume that $h$ satisfies the following properties:

$$
\begin{aligned}
& \qquad h(x) \geq 0 \text { : convex; } \\
& \text { There exists } C>0 \quad \text { such that } h(x) \leq C\left(1+|x|^{n}\right), x \in \mathbb{R},
\end{aligned}
$$

Hindawi Publishing Corporation

Journal of Applied Mathematics and Stochastic Analysis

Volume 2006, Article ID 48369, Pages 1-17

DOI 10.1155/JAMSA/2006/48369 
for some constant $C>0, n \geq 0$. We refer to [5] for the quadratic case of degenerate diffusions related to Riccati equations in case of $h(x)=C x^{2}$ and $n=2$ with infinite horizon.

The purpose of this paper is to show the existence of a viscosity solution of $u$ of the associated Hamilton-Jacobi-Bellman (in short, HJB) equation of the form:

$$
-\beta u+\frac{1}{2} \sigma^{2} x^{2} u^{\prime \prime}+A x u^{\prime}+\min _{r \in \mathbb{R}}\left(r^{2}+r u^{\prime}\right)+h(x)=0 \quad \text { in } \mathbb{R} .
$$

Our method consists in finding a unique viscosity solution for $u$ of (1.5) following Bardi and Capuzzo-Dolcetta [2], Crandall et al. [4], Fleming and Soner [7] through the limit of the solution $v=v_{L}, L>0$, to the HJB equation

$$
-\beta v+\frac{1}{2} \sigma^{2} x^{2} v^{\prime \prime}+A x v^{\prime}+\min _{|r| \leq L}\left(r^{2}+r v^{\prime}\right)+h(x)=0 \quad \text { in } \mathbb{R},
$$

as $L \rightarrow \infty$. To show the existence of the viscosity solution $v_{L}$, we assume that $h$ has the following property: there exists $C_{\rho}>0$, for any $\rho>0$, such that

$$
|h(x)-h(y)| \leq C_{\rho}|x-y|^{n}+\rho\left(1+|x|^{n}+|y|^{n}\right), \quad \forall x, y \in \mathbb{R}
$$

for a fixed integer $n \geq 2$.

This condition acts as the uniform continuity of $h$ with order $n$, and plays an important role for the existence of viscosity solutions $[10,11]$. We notice that (1.7) is fulfilled for $h(x)=|x|^{\bar{n}}, \bar{n} \in[2, \mathbf{n}]$.

In Section 2 we show that the value function $v_{L}(x)=\inf _{c \in \mathscr{A}_{L}} J(c)$ is a unique viscosity solution of (1.6), where $\mathscr{A}_{L}=\left\{c=\left(c_{t}\right) \in \mathscr{A}:\left|c_{t}\right| \leq L\right.$ for all $\left.t \geq 0\right\}$. Section 3 is devoted to the study of $u$ that $u(x):=\lim _{L \rightarrow \infty} v_{L}(x)$ is a viscosity solution of (1.5).

\section{Viscosity solutions}

We here study the properties of the value function $v_{L}(x)$ and show that $v_{L}(x)$ is a viscosity solution of the Bellman equation (1.6) for any fixed $L>0$, and then $v_{L}$ converges to a viscosity solution $u$ of the Bellman equation (1.5).

Given a continuous and degenerate elliptic map $H: \mathbb{R} \times \mathbb{R} \times \mathbb{R} \times \mathbb{R} \rightarrow \mathbb{R}$, we recall by [4] the definition of viscosity solutions of

$$
H\left(x, w, w^{\prime}, w^{\prime \prime}\right)=0 \quad \text { in } \mathbb{R} .
$$

Definition 2.1. $w \in C(\mathbb{R})$ is called a viscosity subsolution (resp., supersolution) of (2.1) if, whenever for $\varphi \in C^{2}(\mathbb{R}), w-\varphi$ attains its local maximum (resp., minimum) at $x \in \mathbb{R}$, then

$$
\begin{gathered}
H\left(x, w(x), \varphi^{\prime}(x), \varphi^{\prime \prime}(x)\right) \leq 0, \\
\text { resp., } H\left(x, w(x), \varphi^{\prime}(x), \varphi^{\prime \prime}(x)\right) \geq 0 .
\end{gathered}
$$


Also $w \in C(\mathbb{R})$ is called a viscosity solution of $(2.1)$ if it is both viscosity sub- and supersolution of (2.1).

According to Crandall et al. [4] and Fleming and Soner [7] this definition is equivalent to the following: for any $x \in \mathbb{R}$,

$$
\begin{array}{ll}
H(x, w(x), p, q) \leq 0 & \text { for }(p, q) \in J^{2,+} w(x), \\
H(x, w(x), p, q) \geq 0 & \text { for }(p, q) \in J^{2,-} w(x),
\end{array}
$$

where $J^{2,+}$ and $J^{2,-}$ are the second-order superjets and subjets defined by

$$
\begin{aligned}
& J^{2,+} w(x)=\left\{(p, q) \in \mathbb{R}^{2}: \limsup _{y \rightarrow x} \frac{w(y)-w(x)-p(y-x)-(1 / 2) q|y-x|^{2}}{|y-x|^{2}} \leq 0\right\}, \\
& J^{2,-} w(x)=\left\{(p, q) \in \mathbb{R}^{2}: \liminf _{y \rightarrow x} \frac{w(y)-w(x)-p(y-x)-(1 / 2) q|y-x|^{2}}{|y-x|^{2}} \geq 0\right\} .
\end{aligned}
$$

In order to obtain the viscosity property of $v_{L}$, we assume that there exists $\beta_{0} \in(0, \beta)$ satisfying

$$
-\beta_{0}+\sigma^{2} n(2 n-1)+2 n|A|<0,
$$

and we set $f_{k}(x)=\gamma+|x|^{k}$ for any $2 \leq k \leq 2 n$ and a constant $\gamma \geq 1$ chosen later.

\subsection{Properties of viscosity solutions}

Lemma 2.2. Assume (2.6). Then there exist $\gamma \geq 1$ and $\eta>0$, depending on $L$, $k$, such that

$$
-\beta_{0} f_{k}+\frac{1}{2} \sigma^{2} x^{2} f_{k}^{\prime \prime}+A x f_{k}^{\prime}+\max _{|r| \leq L}\left(r^{2}+r f_{k}^{\prime}\right)+\eta f_{k} \leq 0 .
$$

Further

$$
\begin{gathered}
E\left[\int_{0}^{\tau} e^{-\beta_{0} s} \eta f_{k}\left(x_{s}\right) d s+e^{-\beta_{0} \tau} f_{k}\left(x_{\tau}\right)\right] \leq f_{k}(x) \quad \text { for } 2 \leq k \leq 2 n, \\
E\left[\sup _{t} e^{-\beta_{0} t} f_{k}\left(x_{t}\right)\right]<\infty \quad \text { for } 2 \leq k \leq n,
\end{gathered}
$$

where $\tau$ is any stopping time and $x_{t}$ is the response to $\left(c_{t}\right) \in \mathscr{A}_{L}$.

Proof. By (2.6), we choose $\eta \in\left(0, \beta_{0}\right)$ such that

$$
-\beta_{0}+\frac{1}{2} \sigma^{2} k(k-1)+k|A|+\eta<0,
$$


4 Viscosity solution for degenerate diffusions

and then $\gamma \geq 1$ such that

$$
\left(-\beta_{0}+\frac{1}{2} \sigma^{2} k(k-1)+k|A|+\eta\right)|x|^{k}+L k|x|^{k-1}+\left(L^{2}+\eta \gamma-\beta_{0} \gamma\right) \leq 0
$$

Then (2.7) is immediate. By (2.7) and Ito's formula, we deduce (2.8). Moreover, by moment inequalities for martingales we get

$$
\begin{aligned}
E\left[\sup _{t} e^{-\beta_{0} t} f_{k}\left(x_{t}\right)\right] & \leq f_{k}(x)+E\left[\sup _{t}\left|\int_{0}^{t} e^{-\beta_{0} s} f_{k}^{\prime}\left(x_{s}\right) \sigma x_{s} d w_{s}\right|\right] \\
& \leq f_{k}(x)+K E\left[\left(\int_{0}^{\infty} e^{-2 \beta_{0} s} \sigma^{2}\left|x_{s}\right|^{2 k} d s\right)^{1 / 2}\right]
\end{aligned}
$$

for some constant $K>0$. Therefore (2.9) follows from this relation together with (2.8).

Theorem 2.3. Assume (1.3), (1.4), (1.7), and (2.6). Then

$$
v_{L} \quad \text { fulfills }(1.3),(1.4),(1.7) \text {, }
$$

and the dynamic programming principle holds, that is,

$$
v_{L}(x)=\inf _{c \in \mathscr{A}_{L}} E\left[\int_{0}^{\tau} e^{-\beta t}\left\{h\left(x_{t}\right)+\left|c_{t}\right|^{2}\right\} d t+e^{-\beta \tau} v_{L}\left(x_{\tau}\right)\right]
$$

for any stopping time $\tau$.

Proof. We supress $L$ of $v_{L}$ for simplicity. The convexity of $v$ follows from the same line as [5, Chapter 4, Lemma 10.6]. Let $x_{t}^{0}$ be the unique solution of

$$
d x_{t}^{0}=A x_{t}^{0} d t+\sigma x_{t}^{0} d w_{t}, \quad x_{0}^{0}=x
$$

Then, by (1.4) and (2.8)

$$
v(x) \leq E\left[\int_{0}^{\infty} e^{-\beta t} h\left(x_{t}^{0}\right) d t\right] \leq C E\left[\int_{0}^{\infty} e^{-\beta_{0} t} f_{n}\left(x_{t}^{0}\right) d t\right] \leq \frac{C f_{n}(x)}{\eta} .
$$

For the solution $y_{t}$ of (1.2) with $y_{0}=y$, it is clear that $x_{t}-y_{t}$ fulfills (2.15) with initial condition $x-y$. We note by (2.10) with $k=n$ and Ito's formula that

$$
E\left[e^{-\beta_{0} t}\left|x_{t}^{0}\right|^{n}\right] \leq|x|^{n}
$$


Thus by (1.7) and (2.8)

$$
\begin{aligned}
|v(x)-v(y)| & \leq \sup _{c \in \mathscr{A}_{L}} E\left[\int_{0}^{\infty} e^{-\beta t}\left|h\left(x_{t}\right)-h\left(y_{t}\right)\right| d t\right] \\
& \leq \sup _{c \in \mathscr{A}_{L}} E\left[\int_{0}^{\infty} e^{-\beta t}\left\{C_{\rho}\left|x_{t}-y_{t}\right|^{n}+\rho\left(1+\left|x_{t}\right|^{n}+\left|y_{t}\right|^{n}\right)\right\} d t\right] \\
& \leq \sup _{c \in \mathscr{A}_{L}} \int_{0}^{\infty} e^{-\beta t}\left\{C_{\rho}|x-y|^{n} e^{\beta_{0} t}+\rho\left(h_{n}(x)+h_{n}(y)\right) e^{\beta_{0} t}\right\} d t \\
& \leq \frac{1}{\beta-\beta_{0}}\left[C_{\rho}|x-y|^{n}+2 \rho \gamma\left(1+|x|^{n}+|y|^{n}\right)\right] .
\end{aligned}
$$

Therefore we get (2.13).

To prove (2.14), we denote by $v^{r}(x)$ the right-hand side of (2.14). By the formal Markov property

$$
E\left[\int_{\tau}^{\infty} e^{-\beta t}\left\{h\left(x_{t}\right)+\left|c_{t}\right|^{2}\right\} d t \mid \mathscr{F}_{\tau}\right]=E\left[\int_{0}^{\infty} e^{-\beta(t+\tau)}\left\{h\left(x_{\tau+t}\right)+\left|c_{\tau+t}\right|^{2}\right\} d t \mid \mathscr{F}_{\tau}\right]=e^{-\beta \tau} J_{\widetilde{c}}\left(x_{\tau}\right),
$$

with $\tilde{c}$ equal to $c$ shifted by $\tau$. Thus

$$
\begin{aligned}
J_{c}(x) & =E\left[\int_{0}^{\tau}+\int_{\tau}^{\infty} e^{-\beta t}\left\{h\left(x_{t}\right)+\left|c_{t}\right|^{2}\right\} d t\right] \\
& =E\left[\int_{0}^{\tau} e^{-\beta t}\left\{h\left(x_{t}\right)+\left|c_{t}\right|^{2}\right\} d t\right]+E\left[\int_{\tau}^{\infty} \frac{e^{-\beta t}\left\{h\left(x_{t}\right)+\left|c_{t}\right|^{2}\right\} d t}{\mathscr{F}_{\tau}}\right] \\
& \geq E\left[\int_{0}^{\tau} e^{-\beta t}\left\{h\left(x_{t}\right)+\left|c_{t}\right|^{2}\right\} d t+e^{-\beta \tau} v_{L}\left(x_{\tau}\right)\right] .
\end{aligned}
$$

It is known in $[7,12]$ that this formal argument can be verified, and we deduce $v_{L}(x) \geq$ $v^{r}(x)$.

To prove the reverse inequality, let $\rho>0$ be arbitrary. We set

$$
V_{c}(x)=E\left[\int_{0}^{\infty} e^{-\beta t}\left\{h\left(x_{t}\right)+\left|c_{t}\right|^{2}\right\} d t\right]
$$

By the same calculation as (2.18), there exists $C_{\rho}>0$ such that

$$
\left|V_{c}(x)-V_{c}(y)\right| \leq C_{\rho}|x-y|^{n}+\rho\left(1+|x|^{n}+|y|^{n}\right) .
$$


6 Viscosity solution for degenerate diffusions

Take $0<\delta<1$ with $C_{\rho} \delta^{n}<\rho$. Then, we have for $|x-y|<\delta$,

$$
\begin{aligned}
|v(x)-v(y)| & \leq \sup _{c \in \mathscr{A}_{L}}\left|V_{c}(x)-V_{c}(y)\right| \\
& \leq \rho\left(2+|x|^{n}+|y|^{n}\right) \\
& \leq \rho\left[2+|x|^{n}+2^{n}\left(1+|x|^{n}\right)\right] \\
& =\rho\left[\left(2+2^{n}\right)+\left(1+2^{n}\right)|x|^{n}\right] \\
& \leq \Xi_{\rho}(x):=\rho\left(2^{n}+2\right)\left(1+|x|^{n}\right) .
\end{aligned}
$$

Let $\left\{S_{i}\right\}$ be a sequence of disjoint subsets of $\mathbf{R}$ such that

$$
\operatorname{diam}\left(S_{i}\right)<\delta, \quad \cup_{i} S_{i}=\mathbb{R}
$$

For any $i$, we take $x^{(i)} \in S_{i}$ and $c^{(i)} \in \mathscr{A}_{L}$ such that

$$
V_{c^{(i)}}\left(x^{(i)}\right) \leq \inf _{c \in A_{L}} V_{c}\left(x^{(i)}\right)+\rho .
$$

Define $c^{\tau} \in \mathscr{A}_{L}$ by

$$
c_{t}^{\tau}=c_{t} 1_{\{t<\tau\}}+c_{t-\tau}^{(i)} 1_{\left\{x_{\tau} \in S_{i}\right\}} 1_{\{t \geq \tau\}}, \quad \text { for } x_{\tau} \in S_{i} .
$$

Hence,

$$
\begin{aligned}
V_{c^{(i)}}\left(x_{\tau}\right) & =V_{c^{(i)}}\left(x_{\tau}\right)-V_{c^{(i)}}\left(x^{(i)}\right)+V_{c^{(i)}}\left(x^{(i)}\right) \\
& \leq \Xi_{\rho}\left(x_{\tau}\right)+V_{c^{(i)}}\left(x^{(i)}\right) \\
& \leq \Xi_{\rho}\left(x_{\tau}\right)+\inf _{c \in A_{L}} V_{c}\left(x^{(i)}\right)+\rho \\
& =\Xi_{\rho}\left(x_{\tau}\right)+v\left(x^{(i)}\right)+\rho \\
& \leq 2 \Xi_{\rho}\left(x_{\tau}\right)+v\left(x_{\tau}\right)+\rho .
\end{aligned}
$$

Now, by the definition of $v^{r}(x)$, we can find $c \in \mathscr{A}_{L}$ such that

$$
v^{r}(x)+\rho \geq E\left[\int_{0}^{\tau} e^{-\beta t}\left\{h\left(x_{t}\right)+\left|c_{t}\right|^{2}\right\} d t+e^{-\beta \tau} v\left(x_{\tau}\right)\right] .
$$


Thus, using the formal Markov property [7], we have

$$
\begin{aligned}
v^{r}(x)+\rho \geq & \sum_{i} E\left[\int_{0}^{\tau} e^{-\beta t}\left\{h\left(x_{t}\right)+\left|c_{t}\right|^{2}\right\} d t+e^{-\beta \tau}\left(V_{c^{(i)}}\left(x_{\tau}\right)-2 \Xi_{\rho}\left(x_{\tau}\right)-\rho\right): x_{\tau} \in S_{i}\right] \\
= & E\left[\int_{0}^{\tau} e^{-\beta t}\left\{h\left(x_{t}^{\tau}\right)+\left|c_{t}^{\tau}\right|^{2}\right\} d t+\int_{\tau}^{\infty} e^{-\beta t}\left\{h\left(x_{t}^{\tau}\right)+\left|c_{t}^{\tau}\right|^{2}\right\} d t \mid \mathscr{F}_{\tau}\right] \\
& -2 E\left[e^{-\beta \tau} \Xi_{\rho}\left(x_{\tau}\right)\right]-\rho \geq v(x)-2 \Xi_{\rho}(x)-\rho,
\end{aligned}
$$

where $x_{t}^{\tau}$ is the response to $c_{t}^{\tau}$ with $x_{0}^{\tau}=x_{\tau}$. Letting $\rho \rightarrow 0$, we deduce $v^{r}(x) \geq v(x)$, which completes the proof.

Lemma 2.4. Let $h>0$, then

$$
\begin{gathered}
\sup _{c \in \mathscr{A}_{L}} \sup _{0 \leq t \leq h} E\left|x_{t}\right|^{2}<\infty, \\
\lim _{h \rightarrow 0} \sup _{c \in \mathscr{A}_{L}} E\left[\sup _{0 \leq t \leq h}\left|x_{t}-x\right|^{2}\right]=0 .
\end{gathered}
$$

Proof. By Ito's formula, we have

$$
\begin{aligned}
\left|x_{t}\right|^{2}= & |x|^{2}+\int_{0}^{t}\left\{2 A x_{t}\left|x_{t}\right| \operatorname{sgn}\left(x_{t}\right)+2\left|x_{t}\right| \operatorname{sgn}\left(x_{t}\right) c_{t}+\sigma^{2} x_{t}^{2}\right\} d t \\
& +\int_{0}^{t} 2\left|x_{t}\right| \operatorname{sgn}\left(x_{t}\right) d w_{t} .
\end{aligned}
$$

Let

$$
\tau_{n}= \begin{cases}\inf \left\{t:\left|x_{t}\right|>n\right\}, & \text { if }\left\{t:\left|x_{t}\right|>n\right\} \neq \varnothing \\ \infty, & \text { if }\left\{t:\left|x_{t}\right|>n\right\}=\varnothing\end{cases}
$$

Then

$$
\begin{aligned}
E\left[\left|x_{t \wedge \tau_{n}}\right|^{2}\right]= & |x|^{2}+E\left[\int_{0}^{t \wedge \tau_{n}}\left\{2 A+\sigma^{2}\right\}\left|x_{t}\right|^{2} d t\right]+E\left[\int_{0}^{t \wedge \tau_{n}} 2\left|x_{t}\right| \operatorname{sgn}\left(x_{t}\right) c_{t} d t\right] \\
& +E\left[\int_{0}^{t \wedge \tau_{n}} \sigma x_{t} 2\left|x_{t}\right| \operatorname{sgn}\left(x_{t}\right) d w_{t}\right] \\
\leq & |x|^{2}+\left(2 A+\sigma^{2}\right) E\left[\int_{0}^{t \wedge \tau_{n}}\left|x_{t}\right|^{2} d t\right]+2 L E\left[\int_{0}^{t \wedge \tau_{n}}\left(1+\left|x_{t}\right|^{2}\right) d t\right] \\
\leq & \left(|x|^{2}+2 L h\right)+\left(2 A+\sigma^{2}+2 L\right) E\left[\int_{0}^{t \wedge \tau_{n}}\left|x_{t}\right|^{2} d t\right] \\
\leq & C_{2}+C_{1} \int_{0}^{t} E\left[\left|x_{s \wedge \tau_{n}}\right|^{2}\right] d s,
\end{aligned}
$$


for $C_{1}=\left(2 A+\sigma^{2}+2 L\right), C_{2}=\left(|x|^{2}+2 L h\right)>0$. Set $E\left[\left|x_{t \wedge \tau_{n}}\right|^{2}\right]=\xi(t)$, then $\xi(t) \leq C_{1} \int_{0}^{t} \xi(s) d s+$ $C_{2}$. By Gronwall's lemma, we have $\xi(t) \leq C_{2}\left(1+C_{1} t e^{C_{1} t}\right)=C_{3} e^{C_{4} t}$, for $C_{3}, C_{4}>0$. Hence $E\left[\left|x_{t \wedge \tau_{n}}\right|^{2}\right] \leq C_{3} e^{C_{4} t}$.

By Fatou's lemma, we obtain

$$
E\left[\left|x_{t}\right|^{2}\right] \leq C_{3} e^{C_{4} t}
$$

Therefore, we obtain (2.30).

To prove (2.31), we have by (2.30)

$$
E\left[\int_{0}^{h}\left|x_{t}\right|^{2} d t\right]=\int_{0}^{h} E\left|x_{t}\right|^{2} d t \leq h C_{3} e^{C_{4} h}
$$

and by the moment inequalities for local martingales [9] and Hölder's inequality

$$
E\left[\left\{\left(\sup _{0 \leq t \leq h}\left|\int_{0}^{t} \sigma x_{t} d w_{t}\right|\right)^{2}\right\}\right] \leq C E\left[\left(\int_{0}^{h}\left|x_{t}\right|^{2} d t\right)\right] \leq C\left[\int_{0}^{h} E\left|x_{t}\right|^{2} d t\right]
$$

for some constant $C>0$. Then

$$
\begin{aligned}
E\left[\sup _{0 \leq t \leq h}\left|x_{t}-x\right|^{2}\right] \leq & 3^{2}\left(E\left[\left(\int_{0}^{h}\left|A x_{t}\right| d t\right)^{2}+\left(\int_{0}^{h}\left|c_{t}\right| d t\right)^{2}+\left(\sup _{0 \leq s \leq h}\left|\int_{0}^{s} \sigma x_{t} d w_{t}\right|\right)^{2}\right]\right) \\
\leq & 3^{2}\left(|A|^{2} E\left[\left(\int_{0}^{h}\left|x_{t}\right|^{2} d t\right)\left(\int_{0}^{h} 1^{2 /(2-1)} d t\right)^{2-1}\right]+h^{2} L^{2}\right. \\
& \left.+E\left[\left\{\left(\sup _{0 \leq t \leq h}\left|\int_{0}^{t} \sigma x_{t} d w_{t}\right|\right)^{2}\right\}^{2 / 2}\right]\right) \\
\leq & 3^{2}\left(|A|^{2} h^{2-1} E\left[\int_{0}^{h}\left|x_{t}\right|^{2} d t\right]+h^{2} L^{2}+C \int_{0}^{h} E\left|x_{t}\right|^{2} d t\right) .
\end{aligned}
$$

Hence, we have (2.31).

Theorem 2.5. Assume (1.3), (1.4), (1.7), and (2.6). Then $v_{L}$ is a viscosity solution of (1.6).

Proof. Let $\varphi \in C^{2}(\mathbb{R})$ and let $z$ be the maximizer of $v_{L}-\varphi$ with $v_{L}(z)=\varphi(z)$. Then $(2.14)$ with the constant control $c_{t}=c \in \mathscr{A}_{L}$ gives

$$
\begin{aligned}
\varphi(z) & \leq E\left[\int_{0}^{s} e^{-\beta t}\left\{h\left(x_{t}\right)+|c|^{2}\right\} d t+e^{-\beta s} v\left(x_{s}\right)\right] \\
& \leq E\left[\int_{0}^{s} e^{-\beta t}\left\{h\left(x_{t}\right)+|c|^{2}\right\} d t+e^{-\beta s} \varphi\left(x_{s}\right)\right],
\end{aligned}
$$


where

$$
d x_{t}=\left[A x_{t}+c\right] d t+\sigma x_{t} d w_{t}, \quad x_{0}=z .
$$

We substract $\varphi(z)$ from both sides and apply Ito's formula to obtain

$$
\begin{aligned}
0 & \leq E\left[\int_{0}^{s} e^{-\beta t}\left\{h\left(x_{t}\right)+|c|^{2}\right\} d t+e^{-\beta s} \varphi\left(x_{s}\right)-\varphi(z)\right] \\
& =E\left[\int_{0}^{s} e^{-\beta t}\left\{h\left(x_{t}\right)+|c|^{2}\right\} d t+\left.\int_{0}^{s} e^{-\beta t}\left\{-\beta \varphi+A x \varphi^{\prime}+\varphi^{\prime} c+\frac{1}{2} \sigma^{2} x^{2} \varphi^{\prime \prime}\right\}\right|_{x=x_{t}} d t\right] .
\end{aligned}
$$

Divide by $s$ and let $s \rightarrow 0$ and by dominated convergence theorem,

$$
0 \leq h(z)-\beta \varphi(z)+A z \varphi^{\prime}(z)+\varphi^{\prime}(z) c+|c|^{2}+\frac{1}{2} \sigma^{2} z^{2} \varphi^{\prime \prime}(z)
$$

Thus (2.3) is verified.

Let $O=O_{x}$ be a bounded neighborhood of $x$ and let $\theta=\theta_{x}$ be the exit time of $x_{t}$ from $0_{x}$. Then, by $(2.31)$

$$
P(\theta \leq h) \leq P\left(\sup _{0 \leq s \leq h}\left|x_{s}-x\right| \geq d(x, \partial \bigcirc)\right) \leq\left(E\left[\sup _{0 \leq s \leq h}\left|x_{s}-x\right|^{2}\right]\right)(d(x, \partial \bigcirc))^{-2},
$$

from which

$$
\frac{1}{h} E[h-h \wedge \theta] \leq \frac{1}{h} E[h-\theta: \theta \leq h] \leq P(\theta \leq h) \longrightarrow 0 \quad \text { as } h \longrightarrow 0 .
$$

Let $\varphi \in C^{2}(\mathbb{R})$ and let $z$ be the minimizer of $v_{L}-\varphi$ with $v_{L}(z)=\varphi(z)$. Take $\tau=h \wedge \theta_{z}$ in (2.14). Then by (2.14), there exists $c_{t}^{h}$ with $\left|c_{t}^{h}\right| \leq L$ such that

$$
v_{L}(z)+h^{2} \geq E\left[\int_{0}^{\tau} e^{-\beta t}\left\{h\left(x_{t}^{h}\right)+\left|c_{t}^{h}\right|^{2}\right\} d t+e^{-\beta \tau} v_{L}\left(x_{\tau}^{h}\right)\right]
$$

where

$$
d x_{t}^{h}=\left[A x_{t}^{h}+c_{t}^{h}\right] d t+\sigma x_{t}^{h} d w_{t}, \quad x_{0}^{h}=z .
$$

Hence

$$
\varphi(z) \geq E\left[\int_{0}^{\tau} e^{-\beta t}\left\{h\left(x_{t}^{h}\right)+\left|c_{t}^{h}\right|^{2}\right\} d t+e^{-\beta \tau} \varphi\left(x_{\tau}^{h}\right)\right]-h^{2} .
$$

We substract $\varphi(z)$ from both sides and apply Ito's formula to obtain

$$
\begin{aligned}
0 \geq \frac{1}{h} E[ & \int_{0}^{\tau} e^{-\beta t}\left\{h\left(x_{t}^{h}\right)+\left|c_{t}^{h}\right|^{2}\right\} d t \\
& \left.\quad+\left.\int_{0}^{\tau} e^{-\beta t}\left\{-\beta \varphi(x)+A x \varphi^{\prime}(x)+\varphi^{\prime}(x) c_{t}^{h}+\frac{1}{2} \sigma^{2} x^{2} \varphi^{\prime \prime}(x)\right\}\right|_{x=x_{t}^{h}} d t\right]-h .
\end{aligned}
$$


For any $\rho>0$, by (1.7), (2.30), and (2.31), we have

$$
\begin{aligned}
\sup _{0 \leq t \leq h} E\left[\left|h\left(x_{t}^{h}\right)-h(z)\right|\right] & \leq \sup _{0 \leq t \leq h} E\left[\rho\left(1+\left|x_{t}^{h}\right|^{n}+|z|^{n}\right)+C_{\rho}\left|x_{t}^{h}-z\right|^{n}\right] \\
& \longrightarrow 0 \quad \text { as } h \longrightarrow 0, \rho \longrightarrow 0 .
\end{aligned}
$$

Hence $\lim _{h \rightarrow 0}(1 / h) E\left[\int_{0}^{h} e^{-\beta t} h\left(x_{t}^{h}\right) d t\right]=h(z)$. Set

$$
\left\{h\left(x_{t}^{h}\right)+\left|c_{t}^{h}\right|^{2}\right\}+\left.\left\{-\beta \varphi(x)+A x \varphi^{\prime}(x)+\varphi^{\prime}(x) c_{t}^{h}+\frac{1}{2} \sigma^{2} x^{2} \varphi^{\prime \prime}(x)\right\}\right|_{x=x_{t}^{h}}=h\left(x_{t}\right) .
$$

By (1.4), (2.30), (2.44), and Schwarz's inequality, we obtain

$$
\begin{aligned}
\frac{1}{h}\left|E\left[\int_{0}^{h} h\left(x_{t}\right) d t\right]-E\left[\int_{0}^{h \wedge \theta} h\left(x_{t}\right) d t\right]\right| \\
\quad=\frac{1}{h} E\left[\int_{h \wedge \theta}^{h}\left|h\left(x_{t}\right)\right| d t\right] \\
\quad \leq \frac{1}{h} E\left[\int_{h \wedge \theta}^{h} K\left(1+\left|x_{t}\right|^{n}\right) d t\right] \\
\quad \leq \frac{K}{h} E\left[\left(\int_{h \wedge \theta}^{h} 1 d t\right)^{1 / 2}\left(\int_{0}^{h}\left(1+\left|x_{t}\right|^{n}\right)^{2} d t\right)^{1 / 2}\right] \\
\quad \leq \frac{K}{h}\left(E\left[\int_{h \wedge \theta}^{h} 1 d t\right]\right)^{1 / 2}\left(E\left[\int_{0}^{h}\left(1+\left|x_{t}\right|^{n}\right)^{2} d t\right]\right)^{1 / 2} \\
\quad=K\left(\frac{1}{h} E[h-h \wedge \theta]\right)^{1 / 2}\left(\frac{1}{h} E\left[\int_{0}^{h}\left(1+\left|x_{t}\right|^{n}\right)^{2} d t\right]\right)^{1 / 2} \\
\quad \longrightarrow 0 \quad \text { as } h \longrightarrow 0 .
\end{aligned}
$$

Thus we have

$$
\begin{aligned}
& 0 \geq \liminf _{h \rightarrow \infty} \frac{1}{h} E\left[\int_{0}^{h} e^{-\beta t}\left\{h\left(x_{t}^{h}\right)+\left|c_{t}^{h}\right|^{2}\right\} d t\right. \\
& \left.+\left.\int_{0}^{h} e^{-\beta t}\left\{-\beta \varphi(x)+A x \varphi^{\prime}(x)+\varphi^{\prime}(x) c_{t}^{h}+\frac{1}{2} \sigma^{2} x^{2} \varphi^{\prime \prime}(x)\right\}\right|_{x=x_{t}^{h}} d t\right] \\
& \geq \liminf _{h \rightarrow \infty} \frac{1}{h} E\left[\int_{0}^{h} e^{-\beta t}\left\{h(z)+\left|c_{t}^{h}\right|^{2}\right\} d t\right. \\
& \left.+\left.\int_{0}^{h} e^{-\beta t}\left\{-\beta \varphi(x)+A x \varphi^{\prime}(x)+\varphi^{\prime}(x) c_{t}^{h}+\frac{1}{2} \sigma^{2} x^{2} \varphi^{\prime \prime}(x)\right\}\right|_{x=z} d t\right] \\
& \geq h(z)-\beta \varphi(z)+A z \varphi^{\prime}(z)+\min _{|c| \leq L}\left(|c|^{2}+\varphi^{\prime}(z) c\right)+\frac{1}{2} \sigma^{2} z^{2} \varphi^{\prime \prime}(z),
\end{aligned}
$$

which implies (2.2) and completes the proof. 
Theorem 2.6 (Apostol [1] (Dini)). Suppose that $X$ is a compact metric space and $\left\{v_{L}(x)\right\}$ is a sequence of continuous functions that converges pointwise to a continuous function $u$ on $X$. Suppose also that the sequence is pointwise nonincreasing, that is, for each $x \in X$, there exists $v_{L}(x) \geq v_{L+1}(x)$, for all $L \in \mathbb{N}$. Then $\left\{v_{L}(x)\right\}$ converges uniformly to $u$ on $X$.

2.2. Uniqueness of HJB. The most important feature of the theory of viscosity solution is the powerful uniqueness theorem. In the context of optimal control problems, value function is the unique viscosity solutions.

In this section we give a detailed proof of uniqueness result for the quadratic control problem that $v$ is a unique viscosity solution of (1.6). The references for these uniqueness results are Bardi and Capuzzo-Dolcetta [2] and Flacone and Makridakis [6].

Theorem 2.7. Assume (1.3), (1.4), (1.7), and (2.6). Let $v_{i}(i=1,2)$ be two viscosity solutions of (1.6) satisfying (2.13). Then $v_{1}=v_{2}$.

Proof. We first note (2.6) and there exists $n<k<n+1$ such that

$$
-\beta \psi_{k}+\frac{1}{2} \sigma^{2} x^{2} \psi_{k}^{\prime \prime}+A x \psi_{k}^{\prime}+\min _{r \in \mathbb{R}}\left(|r|^{2}+r \psi_{k}^{\prime}\right) \leq 0
$$

where $\psi_{k}(x)=\left(1+|x|^{k}\right)$. Indeed, by (2.6) we choose $\vartheta \in(0, \beta)$ such that

$$
-\beta+\frac{1}{2} k(k-1) \sigma^{2}+k|A|+\vartheta<0 .
$$

By (2.54), we have

$$
\left(-\beta+\frac{1}{2} k(k-1) \sigma^{2}+k|A|+\vartheta\right)|x|^{k}-\frac{k^{2}}{4}\left(|x|^{k-1}\right)^{2}-\beta-\vartheta|x|^{k}<0 .
$$

Then (2.53) is immediate.

Suppose that $v_{1}\left(x_{0}\right)-v_{2}\left(x_{0}\right)>0$ for some $x_{0} \in \mathbb{R}$. Then we find $\eta>0$ such that

$$
\sup _{x \in \mathbb{R}}\left[v_{1}(x)-v_{2}(x)-2 \eta \psi_{k}(x)\right]>0 .
$$

Since

$$
v_{1}(x)-v_{2}(x)-2 \eta \psi_{k}(x) \leq \bar{K}\left(1+|x|^{n}\right)-2 \eta\left(1+|x|^{k}\right) \longrightarrow-\infty \quad \text { as } x \longrightarrow \infty,
$$

there exists $\bar{x} \in \mathbb{R}$ such that

$$
\sup _{x \in \mathbb{R}}\left[v_{1}(x)-v_{2}(x)-2 \eta \psi_{k}(x)\right]=v_{1}(\bar{x})-v_{2}(\bar{x})-2 \eta \psi_{k}(\bar{x})>0 .
$$

Define

$$
\Phi(x, y)=v_{1}(x)-v_{2}(y)-\frac{m}{2}|x-y|^{2}-\eta\left(\psi_{k}(x)+\psi_{k}(y)\right),
$$


12 Viscosity solution for degenerate diffusions

for any $m>0$. It is clear that

$$
\Phi(x, y) \leq C\left(1+|x|^{n}+|y|^{n}\right)-\eta\left(2+|x|^{k}+|y|^{k}\right) \longrightarrow-\infty \quad \text { as } x, y \longrightarrow \infty,
$$

where $C>\max \{\bar{K}, \rho\}$. Hence we find $\left(x_{m}, y_{m}\right) \in \mathbb{R}^{2}$ such that

$$
\begin{aligned}
\Phi\left(x_{m}, y_{m}\right) & =\sup _{x, y} \Phi(x, y) \\
& =v_{1}\left(x_{m}\right)-v_{2}\left(y_{m}\right)-\frac{m}{2}\left|x_{m}-y_{m}\right|^{2}-\eta\left(\psi_{k}\left(x_{m}\right)+\psi_{k}\left(y_{m}\right)\right) \\
& \geq v_{1}(\bar{x})-v_{2}(\bar{x})-2 \eta \psi_{k}(\bar{x})>0,
\end{aligned}
$$

from which

$$
\begin{aligned}
\frac{m}{2}\left|x_{m}-y_{m}\right|^{2} & <v_{1}\left(x_{m}\right)-v_{2}\left(y_{m}\right)-\eta\left(\psi_{k}\left(x_{m}\right)+\psi_{k}\left(y_{m}\right)\right) \\
& \leq C\left(2+\left|x_{m}\right|^{n}+\left|y_{m}\right|^{n}\right)-\eta\left(2+\left|x_{m}\right|^{k}+\left|y_{m}\right|^{k}\right) \\
& \longrightarrow-\infty \text { as }\left|x_{m}\right|,\left|y_{m}\right| \longrightarrow \infty,
\end{aligned}
$$

where $C>\max \{\bar{K}, \rho\}$. Thus we deduce that the sequences $\left\{x_{m}\right\},\left\{y_{m}\right\}$ are bounded and then $\left\{m\left|x_{m}-y_{m}\right|^{2}\right\}$ is bounded by some constant $C>0$, and

$$
\left|x_{m}-y_{m}\right| \leq\left(\frac{C}{m}\right)^{1 / 2} \longrightarrow 0 \quad \text { as } m \longrightarrow \infty \text {. }
$$

Now, we claim that

$$
\begin{array}{cl}
m\left|x_{m}-y_{m}\right|^{2} \longrightarrow 0 & \text { as } m \longrightarrow \infty, \\
x_{m}, y_{m} \longrightarrow \tilde{x} \quad \text { or } & \tilde{y} \text { as } m \longrightarrow \infty .
\end{array}
$$

Indeed, by the definition of $\left(x_{m}, y_{m}\right)$,

$$
\Phi\left(x_{m}, y_{m}\right) \geq v_{1}\left(x_{m}\right)-v_{2}\left(x_{m}\right)-2 \eta \psi_{k}\left(x_{m}\right) \text {. }
$$

Hence, by (1.7) and (2.61)

$$
\begin{aligned}
\frac{m}{2}\left|x_{m}-y_{m}\right|^{2} & \leq v_{2}\left(x_{m}\right)-v_{2}\left(y_{m}\right)+\eta\left(\psi_{k}\left(x_{m}\right)-\psi_{k}\left(y_{m}\right)\right) \\
& =v_{2}\left(x_{m}\right)-v_{2}\left(y_{m}\right)+\eta\left(\left|x_{m}\right|^{k}-\left|y_{m}\right|^{k}\right) \\
& \leq v_{2}\left(x_{m}\right)-v_{2}\left(y_{m}\right)+\eta(n+1) C^{k-1}\left|x_{m}-y_{m}\right| \\
& \leq C_{\rho}\left|x_{m}-y_{m}\right|^{n}+\rho\left(1+\left|x_{m}\right|^{n}+\left|y_{m}\right|^{n}\right)+\eta(n+1) C^{k-1}\left|x_{m}-y_{m}\right|
\end{aligned}
$$


Letting $m \rightarrow \infty$ and then $\rho \rightarrow 0$, we obtain (2.64). Moreover, by (2.63) we have (2.65) taking a subsequence if necessary. Equation (2.63) implies $\tilde{x}=\tilde{y}$. Passing to the limit in (2.61), we get

$$
v_{1}(\tilde{x})-v_{2}(\tilde{x})-2 \eta\left(1+|\tilde{x}|^{k}\right)>0
$$

We apply Ishii’s lemma below to

$$
\begin{aligned}
& V_{1}(x)=v_{1}(x)-\eta \psi_{k}(x), \\
& V_{2}(y)=v_{2}(y)+\eta \psi_{k}(y) .
\end{aligned}
$$

Lemma 2.8 (Ishii). Let $V_{1},-V_{2}$ be upper semicontinuous in an open domain, and set

$$
\Phi(x, y)=V_{1}(x)-V_{2}(y)-\frac{m}{2}|x-y|^{2} .
$$

Let $(\hat{x}, \hat{y})$ be the local maximizer of $\Phi(x, y)$. Then there exist symmetric matrices $X_{1}, X_{2}$ such that

$$
\begin{gathered}
\left(m(\hat{x}-\hat{y}), X_{1}\right) \in \bar{J}^{2,+} V_{1}(\hat{x}), \\
\left(m(\hat{x}-\hat{y}), X_{2}\right) \in \bar{J}^{2,-} V_{2}(\hat{y}), \\
\left(\begin{array}{cc}
X_{1} & 0 \\
0 & -X_{2}
\end{array}\right) \leq 3 m\left(\begin{array}{cc}
I & -I \\
I & I
\end{array}\right), \quad I=\text { identity, }
\end{gathered}
$$

where

$$
\begin{aligned}
\bar{J}^{2, \pm} V_{1}(x)=\left\{\left(p, X_{1}\right): \exists x_{r} \longrightarrow x, \exists\left(p_{r}, X_{r}\right) \in J^{2, \pm} V_{1}\left(x_{r}\right),\right. \\
\\
\left.\left(V_{1}\left(x_{r}\right), p_{r}, X_{r}\right) \longrightarrow\left(V_{1}(x), p, X_{1}\right)\right\} .
\end{aligned}
$$

Proof. For the proof, see Crandall et al. [4, Theorem 3.2], Fleming and Soner [7, Lemma 6.1, page 238], and Ishii [8, Lemma 1, page 149].

We remark that if $V_{1}, V_{2} \in C^{2}$, then

$$
\Phi_{x}(\hat{x}, \hat{y})=\Phi_{y}(\hat{x}, \hat{y})=0
$$

from which

$$
V_{1}^{\prime}(\hat{x})=m(\hat{x}-\hat{y}), \quad V_{2}^{\prime}(\hat{y})=-m(\hat{x}-\hat{y})
$$

Since

$$
\Phi_{x x}=V_{1}^{\prime \prime}(x)-m, \quad \Phi_{x y}=m, \quad \Phi_{y y}=-V_{2}^{\prime \prime}(y)-m,
$$


the maximum principle gives

$$
0 \geq D^{2} \Phi(\hat{x}, \hat{y})=\left(\begin{array}{cc}
V_{1}^{\prime \prime}(\hat{x}) & 0 \\
0 & -V_{2}^{\prime \prime}(\hat{y})
\end{array}\right)-m\left(\begin{array}{cc}
1 & -1 \\
-1 & 1
\end{array}\right)
$$

We obtain $X_{1}, X_{2} \in \mathbb{R}^{1}$ such that

$$
\begin{gathered}
\left(m\left(x_{m}-y_{m}\right), X_{1}\right) \in \bar{J}^{2,+} V_{1}\left(x_{m}\right), \\
\left(m\left(x_{m}-y_{m}\right), X_{2}\right) \in \bar{J}^{2,-} V_{2}\left(y_{m}\right), \\
-3 m\left(\begin{array}{ll}
I & 0 \\
0 & I
\end{array}\right) \leq\left(\begin{array}{cc}
X_{1} & 0 \\
0 & -X_{2}
\end{array}\right) \leq 3 m\left(\begin{array}{cc}
I & -I \\
-I & I
\end{array}\right), \quad I=\text { identity, }
\end{gathered}
$$

where

$$
\begin{aligned}
& \bar{J}^{2, \pm} V_{i}(x)=\left\{(p, X): \exists x_{r} \longrightarrow x, \exists\left(p_{r}, X_{r}\right) \in J^{2, \pm} V_{i}\left(x_{r}\right),\right. \\
&\left.\left(V_{i}\left(x_{r}\right), p_{r}, X_{r}\right) \longrightarrow\left(V_{i}(x), p, X\right)\right\}, \quad i=1,2 .
\end{aligned}
$$

Recall that

$$
\begin{aligned}
& J^{2,+} v_{1}(x)=\left\{\left(p+\eta k|x|^{k-1} \operatorname{sgn}(x), X+\eta k(k-1)|x|^{k-2}\right):(p, X) \in J^{2,+} V_{1}(x)\right\}, \\
& J^{2,-} v_{2}(y)=\left\{\left(p-\eta k|y|^{k-1} \operatorname{sgn}(y), X-\eta k(k-1)|y|^{k-2}\right):(p, X) \in J^{2,-} V_{2}(y)\right\} .
\end{aligned}
$$

Hence

$$
\begin{aligned}
\left(p_{1}, \bar{X}_{1}\right):= & \left(m\left(x_{m}-y_{m}\right)+\eta k\left|x_{m}\right|^{k-1} \operatorname{sgn}\left(x_{m}\right), X_{1}+\eta k(k-1)\left|x_{m}\right|^{k-2}\right) \\
\in & \bar{J}^{2,+} v_{1}\left(x_{m}\right), \\
\left(p_{2}, \bar{X}_{2}\right):= & \left(m\left(x_{m}-y_{m}\right)-\eta k\left|x_{m}\right|^{k-1} \operatorname{sgn}\left(y_{m}\right), X_{2}-\eta k(k-1)\left|y_{m}\right|^{k-2}\right) \\
& \in \bar{J}^{2,-} v_{2}\left(y_{m}\right), \\
x_{m}^{2} X_{1} \leq & y_{m}^{2} X_{2} .
\end{aligned}
$$

By virtue of (1.6), (2.2) and (2.3) give

$$
\begin{aligned}
& -\beta v_{1}(x)+\frac{1}{2} \sigma^{2} x^{2} \bar{X}_{1}+A x p_{1}-\frac{\left|p_{1}\right|^{2}}{4}+\left.h(x)\right|_{x=x_{m}} \geq 0, \\
& -\beta v_{2}(y)+\frac{1}{2} \sigma^{2} y^{2} \bar{X}_{2}+A y p_{2}-\frac{\left|p_{2}\right|^{2}}{4}+\left.h(y)\right|_{y=y_{m}} \leq 0 .
\end{aligned}
$$


Putting these inequalities together, we get

$$
\begin{aligned}
\beta\left[v_{1}\left(x_{m}\right)-v_{2}\left(y_{m}\right)\right] & \\
\leq & \frac{1}{2} \sigma^{2}\left(x_{m}{ }^{2} \bar{X}_{1}-y_{m}{ }^{2} \bar{X}_{2}\right)+A\left(x_{m} p_{1}-y_{m} p_{2}\right) \\
& -\frac{1}{4}\left(\left(\left|p_{1}\right|\right)^{2}-\left(\left|p_{2}\right|\right)^{2}\right)+h\left(x_{m}\right)-h\left(y_{m}\right) \\
\leq & \frac{1}{2} \sigma^{2} \eta k(k-1)\left[\left|x_{m}\right|^{k}+\left|y_{m}\right|^{k}\right]+A m\left(x_{m}-y_{m}\right)^{2} \\
& +A \eta k\left[\left|x_{m}\right|^{k}+\left|y_{m}\right|^{k}\right] \\
& -\frac{1}{4}\left[\left(\left.\left|m\left(x_{m}-y_{m}\right)+\eta k\right| x_{m}\right|^{k-1} \operatorname{sgn}\left(x_{m}\right) \mid\right)^{2}\right. \\
& \left.\quad-\left(\left.\left|m\left(x_{m}-y_{m}\right)-\eta k\right| y_{m}\right|^{k-1} \operatorname{sgn}\left(y_{m}\right) \mid\right)^{2}\right]+h\left(x_{m}\right)-h\left(y_{m}\right) .
\end{aligned}
$$

Letting $m \rightarrow \infty$, we have

$$
\begin{aligned}
& \beta\left[v_{1}(\tilde{x})-v_{2}(\tilde{x})\right] \\
& \quad \leq 2 \eta\left[\frac{1}{2} \sigma^{2} \tilde{x}^{2} k(k-1)|\tilde{x}|^{k-2}+A \tilde{x} k|\tilde{x}|^{k-1}-\frac{k^{2}}{4}\left(|\tilde{x}|^{k-1}\right)^{2}\right] \leq 2 \eta \beta\left(1+|\tilde{x}|^{k}\right),
\end{aligned}
$$

which follows from (2.53). This is contrary of (2.68), completing the proof.

\section{Stability of viscosity solutions of $u$}

Theorem 3.1. Assume (1.3), (1.4), (1.7), and (2.6). Then $u(x)$ is a viscosity solution of (1.5), which satisfies (1.4) and (1.7).

Proof. Combining Theorems 2.5 and 2.6, we get the assertion by the stability result of Fleming and Soner [7, Lemma 6.2, page 73] as follows.

Let $\varphi \in C^{2}(\mathbb{R})$ and let $z$ be the maximizer of $u-\varphi$ such that $u(z)-\varphi(z)>u(x)-\varphi(x)$ in the closed ball $\bar{B}(z, \delta)$ with radius $\delta$ of $z \neq x$.

By Theorem 2.6, $v_{L}-\varphi$ attains a local maximum at some $z_{L} \in \bar{B}(z, \delta)$. Take a subsequence $\left\{z_{L^{\prime}}\right\}$ of $\left\{z_{L}\right\}$ such that

$$
z_{L^{\prime}} \longrightarrow z^{\prime} \in \bar{B}(z, \delta)
$$

By Theorem 2.6

$$
\left(v_{L^{\prime}}-\varphi\right)\left(z_{L^{\prime}}\right) \longrightarrow(u-\varphi)\left(z^{\prime}\right)
$$

Since

$$
\left(v_{L^{\prime}}-\varphi\right)\left(z_{L^{\prime}}\right)>\left(v_{L^{\prime}}-\varphi\right)(x), \quad x \in \bar{B}(z, \delta),
$$


we have

$$
(u-\varphi)\left(z^{\prime}\right) \geq(u-\varphi)(x), \quad x \in \bar{B}(z, \delta) .
$$

In particular,

$$
(u-\varphi)\left(z^{\prime}\right) \geq(u-\varphi)(z) .
$$

Thus we deduce

$$
z=z^{\prime}
$$

and the convergence of the whole sequence.

Now it follows from Theorem 2.5 that

$$
-\beta v_{L}+\frac{1}{2} \sigma^{2} x^{2} \varphi^{\prime \prime}+A x \varphi^{\prime}+\min _{|c| \leq L}\left(|c|^{2}+c \varphi^{\prime}\right)+\left.h(x)\right|_{x=z_{L}} \geq 0 .
$$

Note that

$$
\min _{|c| \leq L}\left(|c|^{2}+c \zeta\right) \longrightarrow \min _{c}\left(|c|^{2}+c \zeta\right) \text { locally uniformly in } \mathscr{R} \quad \text { as } L \longrightarrow \infty
$$

Letting $L \rightarrow \infty$, we get

$$
-\beta u+\frac{1}{2} \sigma^{2} x^{2} \varphi^{\prime \prime}+A x \varphi^{\prime}+\min _{c}\left(|c|^{2}+c \varphi^{\prime}\right)+\left.h(x)\right|_{x=z} \geq 0 .
$$

Again a similar argument gives the proof for the minimizer of $u-\varphi$, that is,

$$
-\beta u+\frac{1}{2} \sigma^{2} x^{2} \varphi^{\prime \prime}+A x \varphi^{\prime}+\min _{c}\left(|c|^{2}+c \varphi^{\prime}\right)+\left.h(x)\right|_{x=z} \leq 0 .
$$

Therefore, we see that $u$ is a viscosity solution of (1.5) and then by Theorem 2.3, it is clear that $u$ fulfills (1.4) and (1.7).

Stochastic control problem: in general we can further study a stochastic control problem for linear degenerate systems to minimize the discounted expected cost:

$$
J(c)=E\left[\int_{0}^{\infty} e^{-\alpha t}\left\{h\left(x_{t}\right)+\left|c_{t}\right|^{m}\right\} d t\right]
$$

over $c \in \mathscr{A}$ subject to the degenerate stochastic differential equation (1.2) and a continuous function $h$ on $\mathbb{R}$ such that (1.4) and (1.7), in addition

$$
k_{0}|x|^{m}-k_{1} \leq h(x)
$$

for some constants $k_{0}, k_{1}>0$ and for a fixed integer $m \geq 2$. 


\section{Acknowledgment}

I would like to express my sincere gratitude to Professor A.B.M. Abdus Sobhan Miah for his advice, support, and encouragement.

\section{References}

[1] T. M. Apostol, Mathematical Analysis, Addison-Wesley, Massachusetts, 1974.

[2] M. Bardi and I. Capuzzo-Dolcetta, Optimal Control and Viscosity Solutions of Hamilton-JacobiBellman Equations, Systems \& Control: Foundations \& Applications, Birkhäuser Boston, Massachusetts, 1997.

[3] A. Bensoussan, Stochastic Control by Functional Analysis Methods, Studies in Mathematics and Its Applications, vol. 11, North-Holland, Amsterdam, 1982.

[4] M. G. Crandall, H. Ishii, and P.-L. Lions, User's guide to viscosity solutions of second order partial differential equations, Bulletin of the American Mathematical Society. New Series 27 (1992), no. 1, 1-67.

[5] G. Da Prato, Direct solution of a Riccati equation arising in stochastic control theory, Applied Mathematics and Optimization 11 (1984), no. 3, 191-208.

[6] M. Falcone and C. Makridakis (eds.), Numerical Methods for Viscosity Solutions and Applications, Series on Advances in Mathematics for Applied Sciences, vol. 59, World Scientific, New Jersey, 2001.

[7] W. H. Fleming and H. M. Soner, Controlled Markov Processes and Viscosity Solutions, Applications of Mathematics (New York), vol. 25, Springer, New York, 1993.

[8] H. Ishii, Viscosity solutions to nonlinear partial differential equations, Sūgaku 46 (1994), no. 2, 144-157 (Japanese).

[9] I. Karatzas and S. E. Shreve, Brownian Motion and Stochastic Calculus, Graduate Texts in Mathematics, vol. 113, Springer, New York, 1988.

[10] S. Koike and H. Morimoto, Variational inequalities for leavable bounded-velocity control, Applied Mathematics and Optimization 48 (2003), no. 1, 1-20.

[11] J.-L. Menaldi and M. Robin, On some cheap control problems for diffusion processes, Transactions of the American Mathematical Society 278 (1983), no. 2, 771-802.

[12] M. Nisio, Lectures on Stochastic Control Theory, ISI Lecture Notes, vol. 9, MacMillan, New Delhi, 1981.

Md. Azizul Baten: Department of Statistics, Shahjalal University of Science and Technology,

Sylhet-3114, Bangladesh

E-mail address: baten@mail.sust.com 


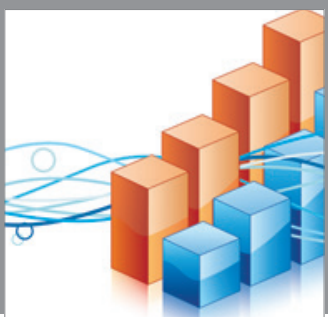

Advances in

Operations Research

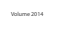

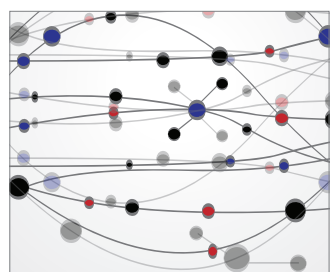

\section{The Scientific} World Journal
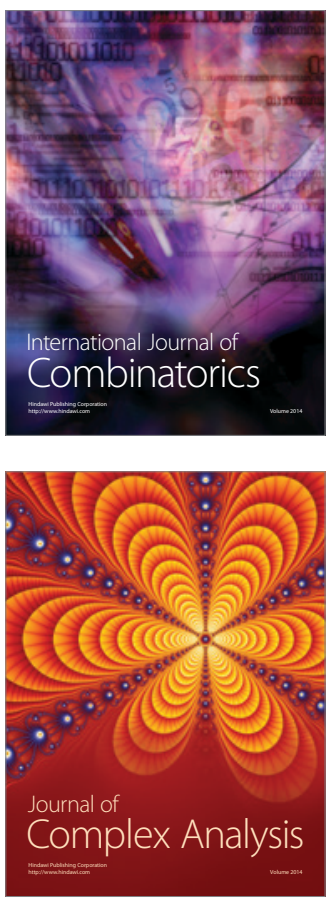

International Journal of

Mathematics and

Mathematical

Sciences
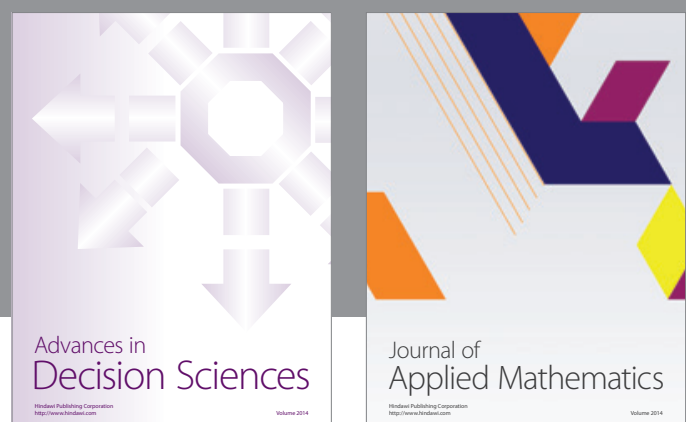

Journal of

Applied Mathematics
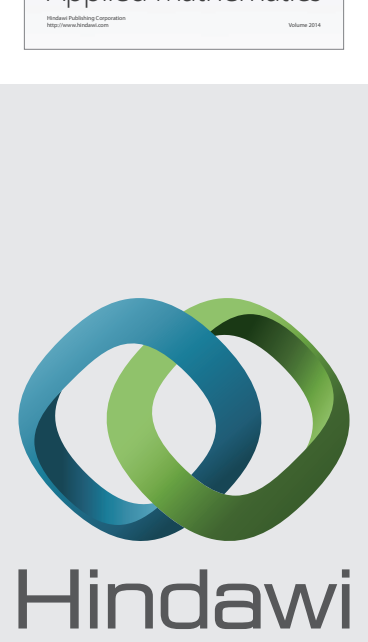

Submit your manuscripts at http://www.hindawi.com
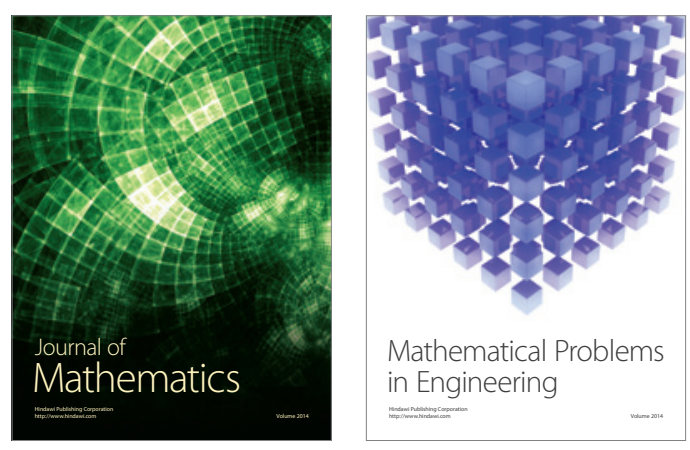

Mathematical Problems in Engineering
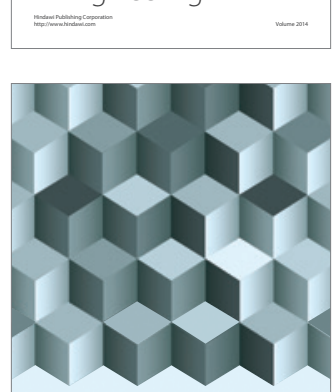

Journal of

Function Spaces
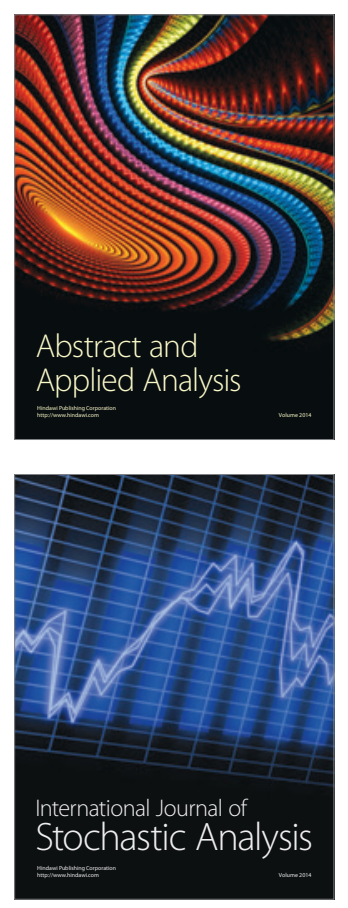

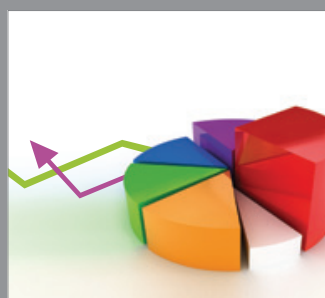

ournal of

Probability and Statistics

Promensencen
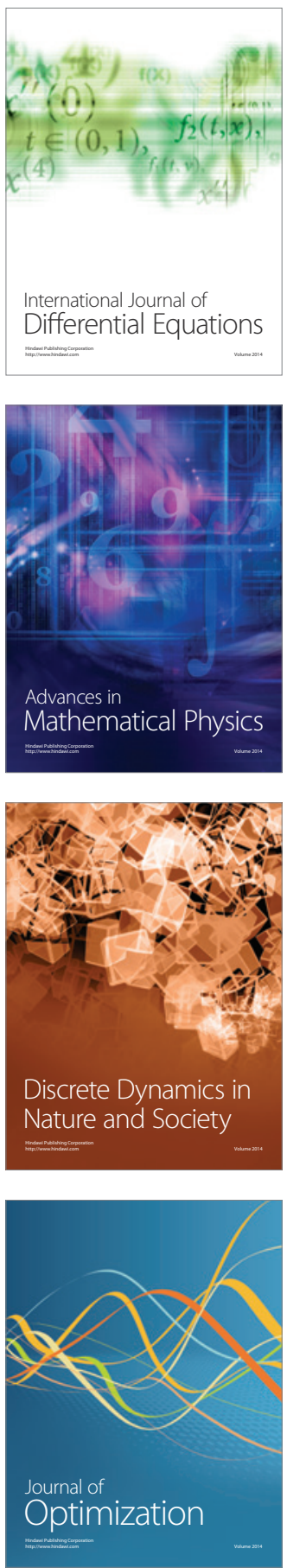